\title{
Mechanisms of Action of Indigenous Antidiabetic Plants from the Boreal Forest of Northeastern Canada
}

\author{
Hoda M. Eid ${ }^{1,2,3}$ and Pierre S. Haddad ${ }^{1,2}$ \\ ${ }^{1}$ Natural Health Products and Metabolic Diseases Laboratory, Department of Pharmacology, Université de Montréal, \\ P.O. Box 6128, Downtown Station, Montreal, QC, Canada H3C 3J7 \\ ${ }^{2}$ Canadian Institutes of Health Research Team in Aboriginal Antidiabetic Medicines and Montreal Diabetes Research Center, \\ Université de Montréal, P.O. Box 6128, Downtown Station, Montreal, QC, Canada \\ ${ }^{3}$ Department of Pharmacognosy, University of Beni-Suef, Beni-Suef 62511, Egypt
}

Correspondence should be addressed to Pierre S. Haddad; pierre.haddad@umontreal.ca

Received 13 May 2014; Revised 9 July 2014; Accepted 14 July 2014; Published 10 August 2014

Academic Editor: Massimiliano Ruscica

Copyright (C) 2014 H. M. Eid and P. S. Haddad. This is an open access article distributed under the Creative Commons Attribution License, which permits unrestricted use, distribution, and reproduction in any medium, provided the original work is properly cited.

Indigenous populations in Canada possess a wealth of native traditional knowledge. However, their rates of Type 2 diabetes mellitus (T2DM), a disease that was unheard of in their midst 50 years ago, are the highest in the country. In an effort to cut the impact of T2DM epidemic on Indigenous health, the Canadian Institutes of Health Research funded the "CIHR Team in Aboriginal Antidiabetic Medicines (CIHR-TAAM)." The goal was to explore Boreal forest medicinal plants stemming from Indigenous Traditional Medicine to be included in T2DM care. Six out of nine communities of the Cree of Eeyou Istchee (CEI) participated in ethnobotanical studies that resulted in the identification of 17 potential antidiabetic plant species. These species were screened for antidiabetic activities using a platform of in vitro bioassays and in vivo models of T2DM. This paper summarizes results on the 10 most promising plant species, their active constituents, and the mechanisms behind their antidiabetic activities. In addition, potential herb-drug interactions were examined at the level of drug-metabolizing enzymes, notably the cytochrome $\mathrm{P} 450$ family. This review serves as a canvas onto which is discussed the value of Indigenous medicinal plants, future avenues of research, and the ethical approach required in this field.

\section{Introduction}

Over the past decades, the world has witnessed a noticeable surge in the number of diabetes mellitus (DM) cases. According to International diabetes federation (IDF), the disease continues to tighten its grip. By 2035, one person out of ten will be diabetic. Every year, DM causes the death of 5.1 million people. In addition, more than a million lower limb amputations, half a million kidney failures, and 1.5 million cases of blindness occur annually as long-term diabetic complications [1].

The two most common forms of DM are type $1 \mathrm{DM}$ (T1DM) and type $2 \mathrm{DM}$ (T2DM). The latter is diagnosed by increased fasting glycemia $(\geq 7 \mathrm{mM})$, elevated postprandial glycemia $(\geq 11 \mathrm{mM})$, and an augmented hemoglobin AlC $(\geq 6.5 \%)$ [2]. While T1DM is still rare in Indigenous populations worldwide, they endure disproportionately high rates of
T2DM. Indigenous populations of Canada are not exceptions to this rule; the age-standardized rates of T2DM among them being several fold higher than the rest of the country (17.2\% versus $5 \%$, resp.) [3]. In general, Indigenous individuals are diagnosed with T2DM at a younger age and Indigenous women have higher rates of gestational diabetes. Finally, considering that obesity is closely associated with T2DM, Indigenous communities have also registered the highest age-adjusted obesity rates in Canada (37.8\% compared to $23 \%$ for the whole Canadian population). The problem is compounded by First Nations' weak compliance to western medicine, which is attributed, at least partially, to the lack of culturally relevant interventions that take into account the holistic nature of Indigenous traditional medicine and its different concepts of health and illness [4].

To address T2DM in Indigenous communities, the Canadian federal government has funded priority research to 
TABLE 1: Boreal forest plants covered in this review.

\begin{tabular}{lcc}
\hline Latin binomial name & Common name & Cree name \\
\hline Abies balsamea (L.) Mill. & Balsam fir & Innasht \\
Alnus incana subsp. Rugosa (Du Roi) R.T. Clausen & Speckled alder & Atushpi \\
Larix laricina (Du Roi) K. Koch & American Larch & Watnagan \\
Picea glauca (Moench) Voss & White spruce & Minhikw \\
Populus balsamifera L. & Balsam poplar & Mitush \\
Rhododendron groenlandicum (Oeder) Kron \& Judd & Labrador tea & Kachichpukw \\
Rhododendron tomentosum Harmaja ssp. subarcticum & Northern Labrador tea & Wishichipukwsh \\
Sarracenia purpurea L. & Purple pitcher plant & Ayigadash \\
Sorbus decora C.K. (Schneid) & Showy mountain ash & Mushkuminanatikw \\
Vaccinium vitis-idaea L. & Lingonberry & Wishichimna \\
\hline
\end{tabular}

promote evidence-based, community-led, and culturally sensitive activities in the health care system. In 2003, the Canadian Institutes of Health Research (CIHR) funded the CIHRTAAM. This project was carried out in close collaboration with local communities of the CEI in Northern Quebec (James Bay area). An unprecedented and comprehensive research agreement was used to manage data, intellectual property, and several aspects of the collaborative research between Cree communities, their health authority, and three major Canadian research universities. This agreement was detailed and discussed elsewhere [5].

The CIHR-TAAM was instated to improve access to Cree traditional medicine practices, such as healing with medicinal plants, within or alongside standard diabetes care. To this aim, the team adopted a community-based, participatory and evidence-based approach. Novel ethnobotanical surveys were initially used to identify potential antidiabetic plants species of the Boreal forest through their traditional indications for several symptoms of diabetes, such as thirst, blurred vision, fatigue, and frequent urination [6]. Information was collected through personal interviews with Elders and Healers in six out of the nine communities of CEI. Since T2DM is a multifactorial complex disease that involves several organs, plant species were then screened using a comprehensive platform of in vitro bioassays for primary and secondary antidiabetic activities, as well as animal models of the disease. This platform has already been described in detail elsewhere [7].

Briefly, primary antidiabetic potential refers to bioassay activities likely to have hypoglycemic correlates in vivo, through actions on insulin-producing and insulin responsive cells responsible for the regulation of glucose and lipid homeostasis such as those of skeletal muscle, liver, and adipose tissue [7]. On the other hand, secondary antidiabetic activities involve protection against long-term diabetic complications by exerting neuroprotective, antiapoptotic, antiinflammatory, antiglycation, and antioxidant properties [7].

As listed in Table 1, this paper will first present an overview of over ten years' work on the ten most promising Cree plants (in alphabetical order), including their identified active phytochemicals, the observed mechanisms through which they exert their antidiabetic activities, and potential herb-drug interactions. This will provide a canvas on which to secondly discuss outlooks in this important area of endocrinology research.

1.1. Abies balsamea (L.) Mill. A. balsamea, commonly known as balsam fir or Canada balsam (Innasht in Cree) belongs to the pine family (Pinaceae). It is widely distributed in Boreal regions of Canada and northern parts of the United States. The Innu First Nation of Quebec, formerly known as Montagnais, eats the inner bark of the plant [8]. A. balsamea has been traditionally used to combat colds, fever, rheumatism, and respiratory tract infections [9]. During the ethnobotanical survey conducted in 2003, the plant stood out as the third most cited species for the management of diabetes symptoms and complications [6]. The ethanol extract of inner bark significantly enhanced basal and insulinstimulated glucose uptake in cultured skeletal muscle cells and adipocytes [10]. It exerted its action on muscle cells by moderately inhibiting and uncoupling mitochondria (energy depletion; see Figure 1), which activated AMPK, but had mild effects on cell $\mathrm{pH}$ or ATP levels [11].

However, more prominent effects were observed on glucose production and storage in hepatocyte cultures. Two assays were used to screen for the stimulation of hepatic glycogenesis and the suppression of glucose-6phosphatase (G6Pase, the key enzyme of hepatic gluconeogenesis; Figure 1). Each bioassay, respectively, put the plant in the forefront as the first and the second most promising species [12]. Further mechanistic studies revealed enhanced phosphorylation of Akt (protein kinase B) and AMP-activated protein kinase (AMPK). These kinases are reliable indicators of stimulation of the major pathways controlling G6Pase activity in hepatocytes (Figure 1), namely, the insulin-dependent phosphatidylinositol 3-kinase (PI3$\mathrm{K})$ pathway and the insulin-independent AMPK pathway, respectively. On the other hand, stimulation of hepatic glycogen synthase (GS), the enzyme that stores excess glucose as glycogen, was positively correlated with increased phosphorylation of glycogen synthase kinase-3 (GSK-3). GSK3 is a serine/threonine kinase and an inhibitor of glycogen synthesis in liver and muscle. The phosphorylation of this enzyme by the plant extract relieves its inhibitory effect on GS and promotes glycogen synthesis (a mechanism similar to that of insulin in glycogenesis, Figure 1) [12]. This raises the 


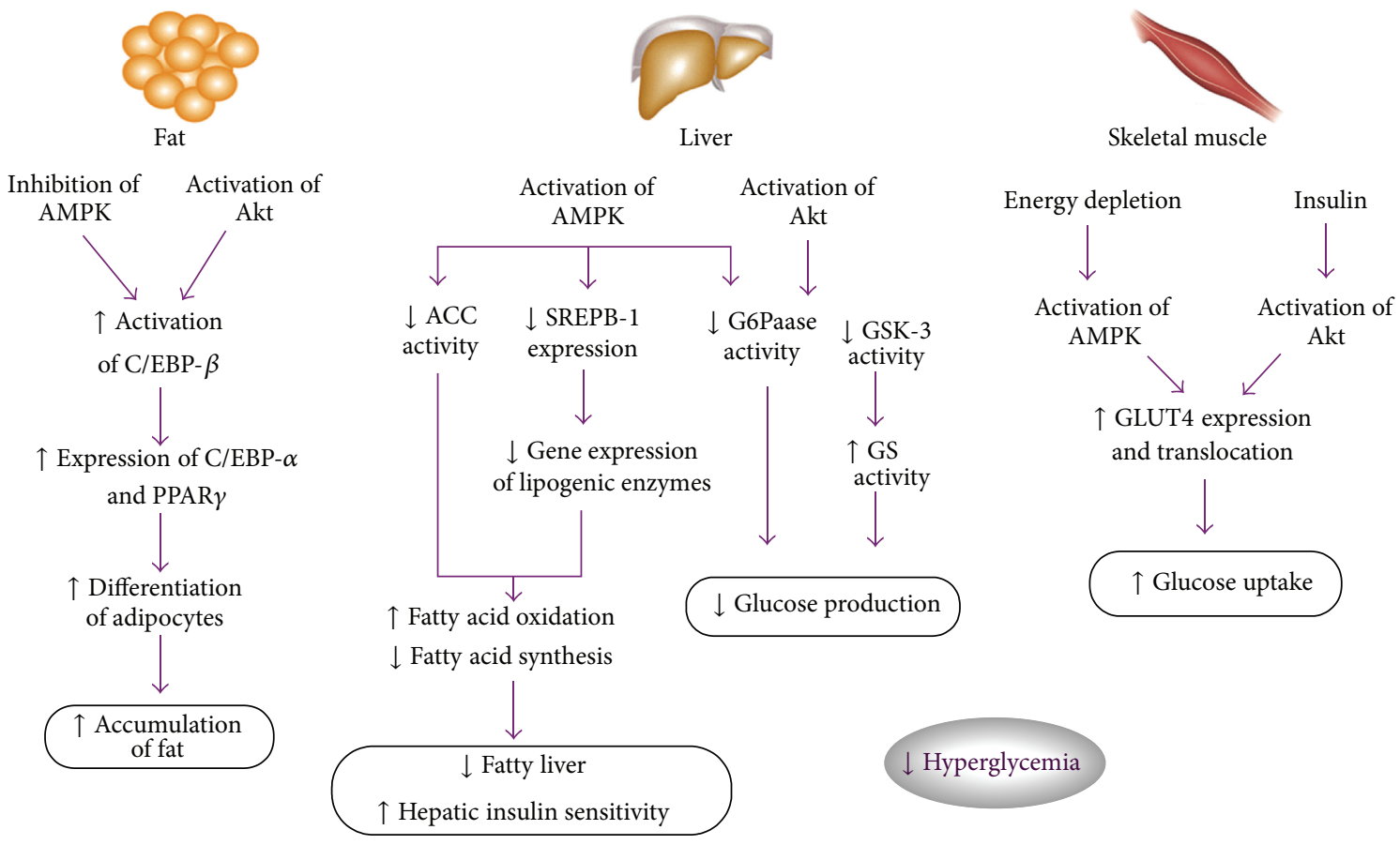

FIGURE 1: Insulin-dependent and -independent regulation of glucidic and lipidic metabolism. In skeletal muscle, insulin and insulinindependent AMPK pathways regulate the translocation of GLUT4 vesicles to plasma membrane that results in stimulation of glucose uptake. In the liver, these pathways act in a concerted fashion to regulate glucose and lipid metabolism. AMPK activation leads to the inhibition of ACC activity thereby inhibiting fatty acid biosynthesis and increasing fatty acid oxidation. Moreover, the suppression of SREPB-1 by AMPK activation inhibits fatty acid synthesis through the downregulation of the lipogenic genes. On the other hand, insulin inhibits hepatic glucose production by suppressing genes encoding gluconeogenic enzymes including G6pase. Concomitantly, insulin enhances the storage of glucose as glycogen via the inhibition of GSK-3, thus relieving its inhibitory action on GS and leads to glycogen synthesis. Finally, insulin and AMPK pathways have opposing roles on adipose tissue metabolism. While Insulin-mediated Akt (PKB) phosphorylation stimulates the differentiation of adipocytes and the accumulation of fat, activation of AMPK has an inhibitory effect. Adipocyte differentiation is mediated, at least in part, through the induction of C/EBP- $\beta$. The latter promotes adipogenesis by upregulating the adipogenic transcription factors $\mathrm{C} / \mathrm{EBP} \alpha$ and PPAR- $\gamma$ gene expression. ACC, acetyl-CoA carboxylase; AMPK, AMP-activated protein kinase; C/EBP, CCAAT-enhancerbinding protein; G6pase, glucose-6-phosphatase; GS, glycogen synthase; GSK-3, glycogen synthase kinase-3; PKB, protein kinase B; PPAR $\gamma$, peroxisome-proliferator-activated receptor gamma; SREPB-1, sterol regulatory element-binding protein.

interesting possibility that GSK-3 may represent a molecular target of antidiabetic plant compounds.

Finally, in terms of potential herb-drug interactions, balsam fir was one of the Cree plants that significantly inhibited the CYP2C19 isoform of recombinant cytochrome P450 [13]. Also in a cell-free system using two different substrates, the plant also showed a tendency toward mechanism-based inhibition (inactivation) of CYP3A4, the cytochrome P450 responsible for the metabolism of nearly $50 \%$ of prescription drugs [14]. Although results in cell-free systems should not be over-interpreted, these studies suggest that the plant should be used cautiously, notably in combination with drugs that have a small therapeutic window or those that are prescribed to diabetics. Indeed, further studies on human liver microsomes (HLM-a higher level of cellular organization than cell-free recombinant enzymes) showed that A. balsamifera was a strong inhibitor of repaglinide metabolism [15]. As with all other Cree plants tested, however, balsam fir neither interfered with gliclazide metabolism, nor with transport of both drugs in CaCo-2 cells, a model epithelium of intestinal origin. This suggests that Cree plants are generally safe in terms of intestinal drug disposition [15].
In summary, balsam fir preparations appear to exert their antidiabetic potential in part by improving muscle and adipose tissue glucose uptake but mostly by reducing hepatic glucose production through both insulin-dependent and insulin-independent mechanisms.

1.2. Alnus incana Subsp. Rugosa (Du Roi) R.T. Clausen. A. incana, also known as Grey Alder or Speckled Alder (Atushpi in Cree), is a deciduous tree of the birch family (Betulaceae) growing in Northeastern North America. The bark is used by Native Americans to treat cutaneous disorders, mouth and gum diseases, and syphilis [16]. It featured among the top 8 most used plants by elders and healers to alleviate symptoms of T2DM in initial ethnobotanical surveys of CEI communities [6]. It was one of the more potent plants to stimulate glucose transport in cultured myocytes and to stimulate AMPK in the latter $[10,17]$. It also inhibited glucose6-phosphatase activity in cultured hepatocytes, albeit without significantly affecting either Akt or AMPK phosphorylation [12]. Finally, it moderately inhibited intestinal glucose absorption [18]. 
However, in a screening assay for adipogenic glitazonelike activity, and to the surprise of the research team, the alcohol extract of $A$. incana bark unexpectedly and strongly prevented the intracellular accumulation of lipid droplets [10]. Subsequent detailed mechanistic studies demonstrated that the plant blocked the differentiation and the maturation of 3T3-L1 preadipocytes (Figure 1) [17]. The antiadipogenic effect of the plant drew attention to its potential antiobesity activities and a bioassay-guided fractionation method was also employed to isolate and identify its active constituents. Using preparative high pressure liquid chromatography (prep-HPLC) for isolation and nuclear magnetic resonance (NMR) spectroscopy to confirm chemical identity, the activity was attributed to oregonin, a diarylheptanoid glycoside, and a minor constituent of the alcohol extract. Although this compound is of frequent occurrence in Alnus species, this study provided the first report of its antiadipogenic activity [17].

A. incana generally exhibited a weak inhibition of most recombinant CYPs tested in a cell-free system [13]. However, the plant's extract did show significant NADPH- and timedependent inhibition of CYP3A4, again in a cell-free system, suggesting that it may act as a mechanism-based inhibitor (inactivation) [14]. Likewise, speckled alder extract was a potent inhibitor of repaglinide metabolism by HLM but not of gliclazide nor of the transport and metabolism of both oral hypoglycaemic drugs across an intestinal epithelium [15]. This suggests that $A$. incana possesses potential for pharmacokinetic interactions with some drugs at the level of the liver and should be used accordingly.

In summary, speckled alder preparations exert some potential antidiabetic activities in cultured muscle, liver, and intestinal cells. However, the plant's most significant action is to exert antiobesity activities by limiting the expansion of adipose tissue; the active component oregonin appears to bear a good part of this activity, at least in vitro.

1.3. Larix laricina (Du Roi) K. Koch. This native North American plant belongs to the larch genus (Larix), family Pinaceae, and has the common name of tamarack or American Larch (Watnagan in Cree). It has been part of healing practices of North American First Nations including the CEI for thousands of years. Inner bark preparations are applied as poultice for infected wounds, burns, and ulcers or used in the form of a herbal tea as a tonic, diuretic, or laxative. Tamarack essential oil is rich in bornyl acetate and is employed as an expectorant and antiseptic. When chewed, resins are helpful in treating indigestion and urinary tract infection [19]. Although not commonly consumed as food, young buds can be eaten raw or cooked [20]. In ethnobotanical studies conducted by our team, this plant ranked third among the top seventeen plants used by CEI communities to treat symptoms of diabetes [21]. The crude extract of L. laricina bark stimulated glucose uptake in cultured skeletal muscle cells while uncoupling and inhibiting mitochondrial function, thereby activating AMPK [17]. Likewise, tamarack extract inhibited liver cell glucose-6-phosphatase by a mechanism involving AMPK phosphorylation [12]. In CaCo-2 cells, L. laricina extract exerted a mild to moderate inhibition of intestinal glucose transport [18].

More importantly, it promoted adipogenesis, a common mechanism of action for the thiazolidinediones antidiabetic agents [10]. In fact, the plant's extract was more powerful than the rosiglitazone control used. It was thus selected as a promising plant species for further studies. Biologically guided fractionation resulted in the isolation and identification of nine compounds based on column chromatography and prep-HPLC as well as infrared and mass spectroscopy. A new triterpenoid and the diterpene labdane derivative 13epitorulosol had the most prominent adipogenic effect (3.7and 2.7-fold increase in accumulation of lipids, resp., when compared to vehicle control) [22]. The newly discovered terpenoid compound was given the common name awashishinic acid in memory of the late Sam Awashish (highly regarded Cree Healer) and in respect for his family, who were and continue being actively involved in the project [22].

Taking it a step further, the antidiabetic potential of $L$. laricina was validated in a mouse model of diet-induced obesity/hyperglycemia. The plant extract was either administered concomitantly with the high fat diet (prevention study) or after the development of obesity and insulin resistance (treatment study). When administered as treatment, the plant was effective at attenuating hyperglycemia, an effect that started at the onset of the treatment but stayed statistically significant with the higher dose only. Furthermore, the higher dose remarkably reduced hyperinsulinemia, which suggests an improvement of insulin resistance. Of note, leptin/adiponectin ratio, another marker of the degree of insulin resistance, was reduced in both prevention and treatment studies, albeit not reaching statistical significance at the lower dose in the prevention study. In agreement with previous data revealing uncoupling of oxidative phosphorylation in isolated rat mitochondria, skin temperature was significantly elevated during the treatment study and in animals that received the higher dose in the prevention study [23]. In light of these findings, one can present L. laricina as a very promising traditional medicinal plant that could be included in diabetes care regimens among CEI communities.

Nonetheless, it should be used with care. Indeed, tamarack extracts strongly inhibited the CYP2C19 isoform of drug-metabolizing enzymes but did not affect CYP3A4 activity $[13,14]$ in cell-free bioassays. In HLM, L. laricina extract and two major stilbenes derived thereof were found to be potent inhibitors of repaglinide but not gliclazide metabolism [15].

In summary, tamarack possesses significant antidiabetic properties both in vitro and in vivo. These actions involve muscle, liver, and adipose tissue to enhance insulin sensitivity through metformin-(activation of liver and muscle AMPK) and glitazone-(enhancement of adipogenesis) like activities.

1.4. Picea glauca (Moench) Voss. P. glauca (white or Canadian spruce; Minhikw in Cree) is another plant of the pine family and a valuable component of the North American Boreal forests. Native American populations have historically used white spruce as food, traditional medicine, and firewood [24]. 
Similar to A. balsamea, the alcohol extract of $P$. glauca needles showed significant activity in cultured hepatocytes. It came out as the top inhibitor of G6Pase. Nonetheless, the weak correlation with activation of either AMPK or Akt suggested that other pathways might be engaged in modulating the activity of G6Pase. On the other hand, stimulation of GS by the extract was insignificant [12]. White spruce extracts were also among seven Cree plants that exerted a powerful dose-dependent inhibition of intestinal glucose transport in $\mathrm{CaCa}-2$ cells. However, this effect may be short-lasting since it waned in longer-term experiments [18].

Importantly, Cree plant extracts were screened for exerting cytoprotective activities on neuronal precursor cells. $P$. glauca induced a prominent protective effect against both glucose toxicity and glucose deprivation [21]. The effect was also found to be dose-dependent and organ-specific as bark extracts had negligible activity, while cone extracts further enhanced effects of high or low glucose. Needle and cone extracts were chemically diverse as assessed by HPLC coupled to a photodiode array detector and an online atmospheric pressure chemical ionization (DAD-APCI) mass spectrometer. They contained phenolic acid, stilbene, and flavonoid derivatives, in contrast to bark extract where only stilbene derivatives were detected. Compounds uniquely detected in $P$. glauca needles warrant further investigation as adjuvant therapy in diabetes neuropathic complications [25].

As with most of other members of the Pinaceae family that were tested, P. glauca significantly inhibited the activity of both recombinant CYP2C9 and CYP2C19 drug-metabolizing enzymes [13]. However, white spruce extracts showed only a nonsignificant tendency to inactivate CYP3A4 in a cell-free system [14]. Likewise, in HLM, the plant extract only mildly to moderately affected the production of major metabolites of repaglinide but left that of gliclazide undisturbed [15].

In summary, white spruce preparations combine interesting properties of inhibiting intestinal glucose transport and liver glucose-6-phosphatase activity while protecting preneuronal cells against high glucose toxicity.

1.5. Populus balsamifera L. Commonly known as balsam poplar (Mitush in Cree), P. balsamifera belongs to the willow plant family (Salicaceae). Balsamifera is derived from a Latin word meaning "balsam bearing" in reference to the tree's sweet-smelling resin or balsam. The plant is native to Boreal and mountain forests in Eastern and Northern parts of North America from Alaska to Labrador and Newfoundland. Resin from buds has long been used in traditional medicine of native North American First Nations for its antiinflammatory properties, to treat sore throats, coughs, chest pain, and rheumatism [26]. Leaves and branches were used in sweat baths to treat rheumatism. The inner bark decoction has been used as a tonic, to treat cold and as eyewash. The inner bark is also used as an emergency food [27].

Aside from $A$. incana discussed above, the ethanol extract of $P$. balsamifera was another plant product that gave an unexpected, and this time complete, inhibition of adipogenesis in the 3T3-L1 cell bioassay [21]. Detailed mechanistic studies demonstrated that $P$. balsamifera exerted this action by intervening in the earliest events of the adipogenetic program; notably, it completely abrogated the process, significantly limited clonal expansion of preadipocytes, and kept cells in a fibroblastic undifferentiated state [17]. The extract also acted as an inhibitor of PPAR- $\gamma$, a key transcription factor controlling the adipogenetic program (Figure 1). The adipogenesis assay was also used to guide the fractionation of the ethanol extract using prep-HPLC for isolation of compounds [28]. NMR spectroscopy then served to identify a single compound responsible for the in vitro antiadipogenic effects of the plant. Salicortin is an ester of salicyl alcohol glucoside and an abundant component of most species of Salix and Populus genera. Together with other salicylates, salicortin plays a major role in plant defense as a feeding deterrent and against different abiotic stresses, including UV radiation [29]. It represents around $1 \%$ of the inner bark mass and $35 \%$ of the mass of the crude alcoholic extract of P. balsamifera [28].

The inhibitory actions of the plant and its active principle on adipogenesis brought to mind an antiobesity potential. Since the research project was focused on antidiabetic activity, the team's researchers asked Cree communities for approval to add the evaluation of antiobesity effects, as required by the research agreement. Approval was granted and the diet induced obese (DIO) mouse model was used to examine the effects of the plant and its active component in vivo. When given at the onset of the high fat diet at 125 and $250 \mathrm{mg} / \mathrm{kg}$ body weight, $P$. balsamifera extract reduced the weight gain observed in DIO controls by nearly $50 \%$ as compared to Chow-fed normal mice. This effect was dose-dependent and accompanied by a decrease of glycemia, insulinemia, hepatic steatosis, and leptin/adiponectin ratio, all indicative of an improved insulin sensitivity (Figure 1). Enhanced energy expenditure was also suggested by higher skin temperature and uncoupling protein-1 (UCP-1) levels. The higher dose also induced a small yet statistically significant reduction in food intake that could not account fully for the reduced weight gain. Hence, $P$. balsamifera crude extract exerted a partial yet significant preventative action against diet induced obesity [30].

A treatment study was then carried out in the same DIO model where mice were allowed to become obese and insulin resistant before the onset of treatment with the plant's crude extract and the corresponding dose of salicortin $(12.5 \mathrm{mg} / \mathrm{kg})$. Both the crude extract and salicortin reduced body weight, glycemia, insulinemia, liver triglycerides, and leptin/adiponectin ratio. However, the profile of activities of the crude extract differed somewhat from that of salicortin. Salicortin's action on body weight gain was immediate and maintained throughout the treatment period whereas the crude extract caused a small initial drop and only began decreasing weight gain significantly after several weeks. In contrast, the crude extract of $P$. balsamifera had a sustained effect on blood glucose whereas salicortin's effects were initially equivalent but waned with time. No less than 25 key proteins in liver, muscle, and fat were probed by Western blot analysis. P. balsamifera extract increased components involved in insulin-responsive fatty acid oxidation in skeletal muscle, liver, and adipose tissue (Figure 1). It also improved 
hepatic and adipose tissue insulin signaling proteins. The effect of salicortin was similar but generally weaker. There were also hints of a reduced inflammatory state, mostly in liver, that is concordant with known effects of plants from the willow family and of the salicylates that they contain [31].

$P$. balsamifera extracts were also quite safe in what pertains to potential herb-drug interactions. Indeed, it had mild effects on all CYPs tested [13] and showed a nonsignificant tendency to alter NADPH- and time-dependent drug metabolism by CYP3A4 [14]. Similarly, in HLM, balsam poplar extract only moderately affected CYP-dependent repaglinide metabolism but spared that of gliclazide as well as glucuronidation reactions [15].

In general, one can thus say that balsam poplar inner bark shows promise as an antiobesity agent. It appears to act by suppressing adipogenesis, by improving insulin resistance and by favouring "energy wastage" mechanisms such as fat oxidation. Salicortin can explain part of the plant's effects, notably on body weight loss but its effect on glucose and lipid homeostasis appears to be weaker and more transient.

1.6. Rhododendron groenlandicum (Oeder) Kron \& Judd. R. groenlandicum, also called Labrador tea or bog tea (Kachichpukw in Cree), is a plant of the Ericaceae family. Indigenous populations including CEI used the leaves to prepare herbal tea to treat asthma, colds, scurvy, fever, and stomach and kidney problems [32]. Recent studies also reported activities such as antiparasitic, antitumor, decongestant, diuretic, sedative, and stomachic [33].

The plant ranked first in the initial ethnobotanical rankings of potential antidiabetic Cree plants [6] and remained second when further ethnobotanical data was added [21]. Following the screening assays for antidiabetic activities in vitro, the plant emerged as a promising species with potential antidiabetic profile in adipocytes, skeletal muscle cells, and hepatocytes [10, 12]. It also exerted moderate to strong inhibition of glucose transport in Caco-2 intestinal cells, an effect that waned with longer incubation times, as with $P$. glauca [18]. Of note, the adipogenic activity of the plant was comparable to that of PPAR- $\gamma$ activator rosiglitazone. Finally, the plant extract triggered a significant suppression of G6Pase activity and concomitant stimulation of AMPK in cultured hepatocytes in vitro (Figure 1) [12].

Using in vitro cell-free assays based on recombinant cytochrome P450 enzymes, Labrador tea alcohol extract showed potent inhibition of the CYP3A4 and CYP2C19 isoforms [13]. However, the same plant extract did not show any tendency for mechanism-based inhibition of CYP3A4 (inactivation [14]). Nonetheless, in HLM, $R$. groenlandicum extract was also a potent inhibitor of repaglinide metabolism, consistent with interference with CYP3A4 activity [15]. When brewed as a hot water decoction, Labrador tea showed an increasing capacity to inhibit CYP3A4 and part of this effect could be related to the presence of quercetin aglycone [34]. Hence, Labrador tea preparations should be used with caution when consumed with prescription drugs, for instance by allowing sufficient time to pass between the intake of each, respectively.
Nevertheless, Labrador tea has an impressive antidiabetic profile by virtue of its actions on muscle, liver, adipose tissue, and intestine in vitro through insulin-dependent and -independent activities.

1.7. Rhododendron tomentosum Harmaja ssp. Subarcticum. Another specie of the genus Rhododendron, commonly known as Marsh Labrador tea or Northern Labrador tea (Wishichipukwsh in Cree), is widely used by Inuit and other Indigenous populations of Canada for the management of respiratory diseases. $R$. tomentosum was the top plant in ethnobotanical studies listing the 17 potential antidiabetic Cree plants [21]. It showed interesting antidiabetic activities in skeletal muscle cells and adipocytes in culture. In addition, it protected against glucose toxicity and glucose deprivation in neuronal precursor cells in vitro [21]. The most abundant constituent is $(+)$-catechin, which accounts for $19 \%$ of the total weight of characterized phenolics. The plant is also rich in other phenolic species including procyanidins and caffeic acid derivatives. Of note, the antioxidant properties of the plant extract correlated positively with its phenolic content [35].

The inhibition of intestinal brush border carbohydrate metabolism constitutes the mechanism of action of the antidiabetic drug acarbose and this reduces intestinal glucose absorption [36]. Interestingly, a screening for inhibition of glucose uptake by intestinal cells in vitro demonstrated a potent dose-dependent inhibitory activity of $R$. tomentosum on intestinal glucose absorption. The effect was coupled to a decreased expression of the $\mathrm{Na}^{+}$-dependent glucose transporter-1 (SGLT1). This transporter is located at the intestinal brush border and mediates the transport of glucose into the intestinal cells above and beyond its concentration gradient. To validate this acute effect in vivo, an oral glucose tolerance test (OGTT) was performed. The plant extract was given orally and 5 minutes later glucose was administered by gastric gavage. $R$. tomentosum significantly reduced the area under the curve of blood glucose levels [18].

Interestingly, a seasonal variation in phenolic constituents as well as antioxidant and anti-inflammatory activities was observed with the ethanol extracts of Northern Labrador tea, which was negatively correlated with daylight hours [35]. The plant also ranked second as the most potent of the 17 Cree plants in terms of ORAC antioxidant activity and of the correlated inhibition of advanced glycation endproduct (AGE) formation [37].

Weaker than its Labrador tea cousin, R. tomentosum was found to moderately to strongly inhibit the CYP3 family of drug-metabolizing enzymes [13]. However, in further cell-free experiments, Northern Labrador tea extract caused significant time-dependent inhibition of CYP3A4, suggesting a potential for mechanism-based inhibition (enzyme inactivation [14]). The plant extract also potently inhibited repaglinide metabolism in HLM, yet improving its intestinal absorption in CaCo-2 cells and leaving HLM gliclazide metabolism intact [15].

In summary, Northern Labrador tea shares some antidiabetic potential with its more southern cousin by improving 
in vitro glucose transport and adipogenesis in cultured myocytes and preadipocytes, respectively. It also protects preneuronal cells in culture against glucose toxicity and prevents AGE formation by its potent antioxidant activity. Interestingly, it was one of the most potent plants to reduce intestinal glucose transport both in vitro and in vivo by reducing the expression of SGLT-1. Hence it combines interesting antidiabetic activities that we have coined as primary (potentially oral hypoglycemic) and secondary (protection against diabetic complications) [7].

1.8. Sarracenia purpurea L. S. purpurea or purple pitcher plant (Ayigadash in Cree) belongs to the Sarraceniaceae family and forms the basis of a marketed pain medication named Sarapin. It has a long history of medicinal use by Native Americans to alleviate several ailments, notably to treat small pox during the 19th century [27].

Similar to other plant species mentioned in the present paper, the alcoholic extract of $S$. purpurea was subjected to antidiabetic screening bioassays. The plant was more potent than metformin at stimulating insulin-dependent glucose uptake in skeletal muscle cells in vitro [10]. Moreover, this effect was later attributed to a metformin-like action on the AMPK pathway (Figure 1) [17]. The extract also demonstrated cytoprotective activity in a neuronal precursor cell model for diabetic neuropathy [10, 37]. In order to identify the active principles, column chromatography and HPLC were used to fractionate the crude plant extract and NMR or HPLC-DAD-APCI mass spectroscopy served to confirm chemical identity. This fractionation process was guided by either the bioassay for stimulation of muscle glucose uptake [35] or for protection against cell death caused by hyper- or hypoglycemic conditions [38]. The first bioassay guided fractionation study resulted in the isolation of 10 known compounds and the new $6^{\prime}$ $\mathrm{O}$-caffeoylgoodyeroside. The following compounds showed significant stimulation of glucose uptake by cultured skeletal muscle cells: $7 \beta$-O-methylmorroniside, rutin, kaempferol-3$O$-rutinoside, kaempferol-3-O-(6" -caffeoylglucoside), morroniside, goodyeroside, and quercetin-3-O-galactoside [35]. Interestingly, quercetin-3-O-galactoside and morroniside emerged again as the active compounds responsible for the cytoprotective effect of $S$. purpurea in neuronal precursor cells [38].

S. purpurea ethanol extract was generally observed to be a weak inhibitor of most recombinant cytochrome P450s tested in a cell-free assay [13]. Similarly, the plant extract did not show any sign of NADPH- or time-dependent inhibition of CYP3A4 [14]. Likewise, in the more complex HLM model, S. purpurea only moderately affected repaglinide metabolism and left that of gliclazide unaffected.

Cree elders told us that the purple pitcher plant should be used cautiously because it was very powerful. Our evidencebased approach in vitro confirmed that the plant is more potent than metformin to stimulate glucose transport in muscle cells and strongly affords protection against diabetic neuropathy.
1.9. Sorbus decora C.K. (Schneid). This member of the rose family (Rosaceae) is commonly known as the showy mountain ash or dogberry (Mushkuminanatikw in Cree) and is native to the Northeastern parts of North America. The ethanolic extract of $S$. decora increased glucose uptake by skeletal muscle cells and protected preneuronal ones against glucotoxicity in vitro [10]. The effect on muscle glucose uptake was related to a metformin-like action involving AMPK activation [17]. S. decora extracts also increased AMPK and GSK-3 phosphorylations in cultured liver cells (Figure 1) [12]. Finally, the plant extract moderately inhibited in vitro intestinal glucose transport in a dose-dependent manner [18].

To validate the effects of the plant in vivo, the extract was administered orally in three models of insulin resistance and diabetes: the streptozotocin type 1 diabetic rat (STZ), the genetic type 2 diabetic mouse $\mathrm{KK}-\mathrm{A}^{\mathrm{y}}$, and the rat rendered insulin resistant with $10 \%$ glucose water consumption for 6 week. The extract was able to lower glycemia and to exert insulin-sensitizing activity in vivo. Insulin sensitivity was inferred from decreased HOMA insulin resistance parameter. This study confirmed the antidiabetic potential and validated CEI traditional medicine [39].

In terms of active principles, three new pentacyclic triterpenes were isolated by silica gel column chromatography and identified by infrared (IR) as well as $1 \mathrm{D}$ and 2D NMR. The fractionation procedure was guided by the stimulation of glucose uptake in cultured skeletal muscle cells [40].

Finally, using recombinant cytochrome P450 isoenzymes in cell-free experiments, the ethanol extract of $S$. decora strongly inhibited several isoforms of the CYP2C (CYP2C8, 9 and 19) and CYP3A (CYP3A4, 5 and 7) families [13]. However, in further experiments with recombinant CYP3A4, the plant did not show any sign of mechanism-based inhibition, suggesting that it rather acts as a reversible inhibitor of this important drug-metabolizing isoenzyme [14]. In HLM, S. decora extract also potently inhibited the CYP3A4-mediated metabolism of repaglinide, yet spared that of gliclazide [15].

Showy mountain ash thus shows another distinct profile of primary and secondary antidiabetic activities by acting on muscle, liver, and intestinal cells on the one hand and preneuronal cells on the other. However, its strong potential to inhibit several drug-metabolizing enzymes in vitro calls for its cautious use in humans.

1.10. Vaccinium vitis-idaea L. V. vitis (family Ericaceae) is commonly known as mountain cranberry or Lingonberry (Wishichimna in Cree). It grows throughout the subarctic, Boreal, and northern temperate regions. In Scandinavian countries, the berries are used to prepare traditional food and herbal remedies. Using in vitro screening bioassays, the berry ethanol extract was the third most potent Cree plant to inhibit hepatic glucose-6-phosphatase, an effect coupled to increased AMPK phosphorylation. V. vitis was also as potent as metformin at enhancing basal or insulin-stimulated glucose uptake in cultured myocytes [21]. Recently, this effect was confirmed to implicate the enhanced translocation of GLUT4 transporters to the myocyte cell surface and was found to involve the activation of AMPK (Figure 1) [41]. 
Fractionation of the extract had previously been carried out using silica gel column chromatography and preparative scale HPLC for isolation and purification while HPLC-DAD-APCI mass spectroscopy served to confirm chemical identity. The fractionation procedure was guided by muscle cell glucose uptake activity and resulted in the isolation and identification of quercetin-3-O-galactoside, quercetin, and quercetin-3-Oglucoside as the active constituents [42]. Similar to the crude berry extract, the active quercetin glycosides and the aglycone also stimulated the AMPK pathway.

To validate the effects of $V$. vitis in vivo, the berry extract was administered to $\mathrm{KKA}^{\mathrm{y}}$ as well as DIO mice. In both models, $V$. vitis extract reduced glycemia and increased skeletal muscle GLUT4 protein expression. In the DIO mouse model, this was accompanied by enhanced Akt and AMPK phosphorylation in both skeletal muscle and liver [43]. In the latter organ the berry extract significantly reduced triglyceride accumulation and hence, hepatic steatosis. In view of the important role that hepatic steatosis plays in mediating systemic insulin resistance [44], these results, along with those of insulin-dependent and -independent pathways, indicate a beneficial effect of $V$. vitis berries on insulin sensitivity (Figure 1). Interestingly, in KK- $\mathrm{A}^{\mathrm{y}}$ animals, body weight was significantly reduced and was associated with a decrease in food intake. Pair-feeding experiments suggested that the effects of $V$. vitis are almost entirely mediated by a putative appetite reducing action ([41], in press). Unlike DIO animals, $\mathrm{KK}-\mathrm{A}^{\mathrm{y}}$ mice have a defect in the Agouti-related peptide (ARP) pathway involved in feeding behaviour and which causes the hyperphagia forming the basis of the diabetic status of this model [45]. This raises the interesting possibility that the appetite reducing action of $V$. vitis berry extract somehow counteracts a disrupted ARP pathway but may not be exhibited when the ARP pathway is intact.

On the other hand, $V$. vitis berry extract appears to be quite innocuous in relation to potential herb-drug interactions. Indeed, the plant extract did not significantly affect the activity of any of the recombinant CYP isoforms tested in cell-free systems [13]. Likewise, it was among the weakest plants when mechanism-based inhibition of CYP3A4 or metabolism of oral hypoglycaemic drugs repaglinide and gliclazide were more specifically examined in a cell-free system [14] or in HLM-based assays [15], respectively.

In summary, mountain cranberry extracts show great promise as a potential antidiabetic regimen to add in Indigenous diabetes care. Indeed, its actions enhance insulindependent and -independent pathways in both muscle and liver, and both in detailed in vitro and in vivo studies. This indicates that $V$. vitis can reduce hepatic glucose production and increase muscle glucose disposition, both of which forecast a positive action to enhance insulin sensitivity in diabetics.

\section{General Outlook regarding Indigenous Traditional Medicine and Diabetes Care}

Indigenous Traditional Medicine in general and medicinal plants in particular are often regarded with great scepticism by the established health authorities, especially in the socalled Western or industrialized world. The present review of the CIHR-TAAM's work over some ten years has hopefully and clearly shown that the application of an evidence-based approach to the study of putative Indigenous antidiabetic plants leads to surprisingly convincing and supportive evidence. Indeed, when we carried out an exercise to prioritize plant species according to the sum of in vivo and in vitro primary and secondary antidiabetic activities, our results corresponded strikingly well with the prioritization carried out by several elders based on Indigenous Traditional Knowledge and worldviews [7]. In fact, our work can humbly be seen as simply translating Indigenous Traditional Knowledge into the scientific language that is better understood by clinical endocrinologists and diabetes researchers.

Indeed, knowing that a given specie can combine metformin- and glitazone-like activities, or a predisposition to reduce intestinal glucose transport with an aptitude to simultaneously prevent diabetic neuropathy, brings a different outlook on Indigenous medicinal plants. One can also bring forth the additional knowledge that several known and novel phytochemicals (flavanoids, terpenes, stilbenes, and so on) are, at least in part, responsible for the pharmacological activities of the plants. This raises interesting perspectives for some form of characterization and quality control of Indigenous traditional preparations. Moreover, several plants show not only pleiotropic biological activities that are highly interesting in themselves but also pleiotropic biological activities that are also synergistic and complementary with that of other plants. This suggests a myriad of potential therapeutic avenues that could rationally combine different plants to correspond with a given diabetic profile. Indeed, T2DM has several metabolic manifestations that could be dealt with in a tailored way, much as multidrug therapy is currently applied. It is, however, recognized that, aside from the potential herbdrug interactions dealt with in our approach, the question regarding the safety of long-term use of such "plant food supplements" needs to be addressed [46]. Thus far, we have administered plant extracts (both hydroalcoholic and aqueous) to mice and rats for subchronic periods ranging from 4 to 8 weeks with no sign of toxicity. Future studies will need to increase the period of use in preclinical studies. In addition, clinical studies will need to document the use of medicinal plants by Indigenous populations and study the impact of their long-term use on glucidic and lipidic homeostasis.

Our team has used labour-intensive and painstaking approaches toward the identification of active principles and of key proteins of insulin-sensitive tissues involved in several plants' beneficial actions. The application of novel highly sensitive and versatile phytochemical methodologies such as quadrupole-time-of-flight (QTOF) mass spectrometry combined with bioinformatic approaches such as principal component and discriminant analyses are very powerful and can identify known and novel compounds in a manner that is complementary to bioassay-guided fractionation. Likewise, instead of carrying out multiple Western blot analyses for numerous proteins in several insulin-sensitive tissues, modern and now more affordable methods of gene expression 
(genechip) technology could be applied to in vivo work. Gene expression profiles and cluster analysis can point researchers toward known or unsuspected pathways to better understand the mode of action of Indigenous medicinal plants. This can even be applied in the clinical setting by using peripheral blood mononucleated cells (PBMC). Although these cells do not strictly represent key insulin responsive elements in the metabolic sense, PBMCs can provide important insight into several cellular mechanisms (e.g., calcium homeostasis and inflammatory pathways) as well as metabolic or hormone responsive pathways.

Finally, it is foreseeable that detractors will raise the spectre of potentially life-threatening herb-drug interactions. Future studies should also extend our team's work toward more complex systems. Moreover, the same modern phytochemical analytical tools can serve to measure both drug and plant metabolites in biological fluids to assess such potential interactions in vivo. Nonetheless, several medicinal plants had quite innocuous profiles on major drug-metabolizing enzymes. On the other hand, clinicians manage drug-drug interactions on a daily basis such that increasing knowledge of the action of medicinal plants on drug metabolism (especially oral hypoglycemics and other drugs commonly prescribed to obese and diabetic patients) will enable a similar management of both drug and herb use in rational therapeutic regimens.

In conclusion, Indigenous traditional medicine should be viewed as an ally rather than a foe, notably when it comes to developing culturally relevant clinical care for Indigenous diabetics themselves. Medicinal plants stemming from the traditional pharmacopeia of various Inuit, First Nation, and Métis traditions actually represent a global heritage that should be protected and studied in an ethical and collaborative way, much as the CIHR-TAAM has strived to do [5]. Not only can traditional plant preparations become complementary therapeutic avenues to consider respectfully but also they may uncover novel molecules to open up an otherwise rather limited pharmaceutical pipeline. Again, respect through internationally accepted access and benefit sharing principles, such as the Bonn guidelines [47] or the Nagoya protocol [48], should be the norm.

\section{Conflict of Interests}

The authors declare that there is no conflict of interests regarding the publication of this paper.

\section{Acknowledgments}

This work was supported by a Team Grant from the Canadian Institutes of Health Research (CTP-79855, CIHR Team in Aboriginal Antidiabetic Medicines) to PSH and was conducted with the consent and support of the Cree Nation of Eeyou Istchee (Eastern James Bay in Quebec, Canada) as well as that of the Cree Board of Health and Social Services of James Bay (Quebec, Canada).

\section{References}

[1] IDF, Diabetes Atlas, IDF, 2013.

[2] A. Soliman, V. DeSanctis, M. Yassin, R. Elalaily, and N. E. Eldarsy, "Continuous glucose monitoring system and new era of early diagnosis of diabetes in high risk groups," Indian Journal of Endocrinology and Metabolism, vol. 18, pp. 274-282, 2014.

[3] V. Douglas, Introduction to Aboriginal Health and Health Care in Canada: Bridging Health and Healing, Springer, 2013.

[4] C. H. Y. Yu and B. Zinman, "Type 2 diabetes and impaired glucose tolerance in aboriginal populations: a global perspective," Diabetes Research and Clinical Practice, vol. 78, no. 2, pp. 159170, 2007.

[5] A. Cuerrier, A. Downing, E. Patterson, and P. Haddad, "Aboriginal antidiabetic plant project with the James Bay Cree of Québec: an insightful collaboration," Journal of Enterprising Communities, vol. 6, no. 3, pp. 251-270, 2012.

[6] C. Leduc, J. Coonishish, P. Haddad, and A. Cuerrier, "Plants used by the Cree Nation of Eeyou Istchee (Quebec, Canada) for the treatment of diabetes: a novel approach in quantitative ethnobotany," Journal of Ethnopharmacology, vol. 105, no. 1-2, pp. 55-63, 2006.

[7] P. S. Haddad, L. Musallam, L. C. Martineau et al., "Comprehensive evidence-based assessment and prioritization of potential antidiabetic medicinal plants: a case study from Canadian eastern James Bay Cree traditional medicine," Evidence-Based Complementary and Alternative Medicine, vol. 2012, Article ID 893426, 14 pages, 2012.

[8] N. J. Turner, Traditional Plant Foods of Canadian Indigenous Peoples Nutrition, Botany and Use, Gordon and Breach Publishers, 2009.

[9] J. W. Herrick and D. R. Snow, Iroquois Medical Botany, Syracuse University Press, 1995.

[10] D. C. A. Spoor, L. C. Martineau, C. Leduc et al., "Selected plant species from the Cree pharmacopoeia of northern Quebec possess anti-diabetic potential," Canadian Journal of Physiology and Pharmacology, vol. 84, no. 8-9, pp. 847-858, 2006.

[11] L. C. Martineau, D. C. A. Adeyiwola-Spoor, D. Vallerand, A. Afshar, J. T. Arnason, and P. S. Haddad, "Enhancement of muscle cell glucose uptake by medicinal plant species of Canada's native populations is mediated by a common, metformin-like mechanism," Journal of Ethnopharmacology, vol. 127, no. 2, pp. 396-406, 2010.

[12] A. Nachar, D. Vallerand, L. Musallam et al., "The action of antidiabetic plants of the Canadian James bay Cree traditional pharmacopeia on key enzymes of hepatic glucose homeostasis," Evidence-Based Complementary and Alternative Medicine, vol. 2013, Article ID 189819, 9 pages, 2013.

[13] T. W. Tam, R. Liu, J. T. Arnason et al., "Actions of ethnobotanically selected Cree anti-diabetic plants on human cytochrome P450 isoforms and flavin-containing monooxygenase 3," Journal of Ethnopharmacology, vol. 126, no. 1, pp. 119-126, 2009.

[14] T. W. Tam, R. Liu, J. T. Arnason et al., "Cree antidiabetic plant extracts display mechanism-based inactivation of CYP3A4," Canadian Journal of Physiology and Pharmacology, vol. 89, no. 1, pp. 13-23, 2011.

[15] C. Cieniak, R. Liu, A. Fottinger et al., "In vitro inhibition of metabolism but not transport of gliclazide and repaglinide by Cree medicinal plant extracts," Journal of Ethnopharmacology, vol. 150, no. 3, pp. 1087-1095, 2013. 
[16] J. K. Crellin and A. L. Tommie Bass, Herbal Medicine Past and Present: A Reference Guide to Medicinal Plants, Duke University Press, 1997.

[17] L. C. Martineau, J. Hervé, A. Muhamad et al., "Anti-adipogenic activities of Alnus incana and Populus balsamifera bark extracts, part I: sites and mechanisms of action," Planta Medica, vol. 76, no. 13, pp. 1439-1446, 2010.

[18] L. A. Nistor Baldea, L. C. Martineau, A. Benhaddou-Andaloussi, J. T. Arnason, É. Lévy, and P. S. Haddad, "Inhibition of intestinal glucose absorption by anti-diabetic medicinal plants derived from the James Bay Cree traditional pharmacopeia," Journal of Ethnopharmacology, vol. 132, no. 2, pp. 473-482, 2010.

[19] B. Gray, "Tamarack (Larch) Larix laricina," in The Boreal Herbal: Wild Food and Medicine Plants of the North, Aroma Borealis Press, 2011.

[20] D. Johnson, L. Kershaw, A. MacKinnon, and J. Pojar, Plants of the Western Boreal Forest and Aspen Parkland, Lone Pine Publishing and the Canadian Forest Service, 1995.

[21] D. Harbilas, L. C. Martineau, C. S. Harris et al., "Evaluation of the antidiabetic potential of selected medicinal plant extracts from the Canadian boreal forest used to treat symptoms of diabetes: part II," Canadian Journal of Physiology and Pharmacology, vol. 87, no. 6, pp. 479-492, 2009.

[22] N. Shang, J. A. Guerrero-Analco, L. Musallam et al., "Adipogenic constituents from the bark of Larix laricina du Roi (K. Koch; Pinaceae), an important medicinal plant used traditionally by the Cree of Eeyou Istchee (Quebec, Canada) for the treatment of type 2 diabetes symptoms," Journal of Ethnopharmacology, vol. 141, no. 3, pp. 1051-1057, 2012.

[23] D. Harbilas, D. Vallerand, A. Brault et al., "Larix laricina, an antidiabetic alternative treatment from the Cree of Northern Quebec pharmacopoeia, decreases glycemia and improves insulin sensitivity in vivo," Evidence-Based Complementary and Alternative Medicine, vol. 2012, Article ID 296432, 10 pages, 2012.

[24] B. Gray, "Spruce Picea glauca (white spruce) Picea mariana (black spruce)," in The Boreal Herbal: Wild Food and Medicine Plants of the North, pp. 259-264, Aroma Borealis Press, 2011.

[25] C. S. Harris, J. Lambert, A. Saleem et al., "Antidiabetic activity of extracts from needle, bark, and cone of Picea glauca: organ-specific protection from glucose toxicity and glucose deprivation," Pharmaceutical Biology, vol. 46, no. 1-2, pp. 126134, 2008.

[26] V. J. Vogel, American Indian Medicine, University of Oklahoma Press, 2013.

[27] D. Moerman, Native American Ethnobotany, Timber Press, Portland, Ore, USA, 1998.

[28] L. C. Martineau, A. Muhammad, A. Saleem et al., "Antiadipogenic activities of Alnus incana and Populus balsamifera bark extracts. Part II: bioassay-guided identification of actives salicortin and oregonin," Planta Medica, vol. 76, no. 14, pp. 15191524, 2010.

[29] F. Chen, C. Liu, T. J. Tschaplinski, and N. Zhao, "Genomics of secondary metabolism in populus: interactions with biotic and abiotic environments," Critical Reviews in Plant Sciences, vol. 28, no. 5, pp. 375-392, 2009.

[30] D. Harbilas, A. Brault, D. Vallerand et al., "Populus balsamifera L. (Salicaceae) mitigates the development of obesity and improves insulin sensitivity in a diet-induced obese mouse model," Journal of Ethnopharmacology, vol. 141, no. 3, pp. 10121020, 2012.
[31] D. Harbilas, D. Vallerand, A. Brault et al., "Populus balsamifera extract and its active component salicortin reduce obesity and attenuate insulin resistance in a diet-induced obese mouse model," Evidence-based Complementary and Alternative Medicine, vol. 2013, Article ID 172537, 13 pages, 2013.

[32] S. Foster and J. A. Duke, A Field Guide to Medicinal Plants: Eastern and Central North America, Peterson Field Guide Series, Houghton Mifflin, 1990.

[33] C. Chartier, H. Staub, and P. Goetz, "Thé du Labrador (lédon du Groenland) (Ledum groenlandicum Oeder)," Phytotherapie, vol. 3, no. 2, pp. 84-87, 2005.

[34] T. W. Tam, R. Liu, A. Saleem et al., "The effect of Cree traditional medicinal teas on the activity of human cytochrome P450mediated metabolism," Journal of Ethnopharmacology, vol. 155, no. 1, pp. 841-846, 2014.

[35] P. Black, A. Saleem, A. Dunford et al., "Seasonal variation of phenolic constituents and medicinal activities of northern labrador tea, Rhododendron tomentosum ssp subarcticum, an Inuit and cree first nations traditional medicine," Planta Medica, vol. 77, no. 14, pp. 1655-1662, 2011.

[36] A. J. Krentz, R. E. Ferner, and C. J. Bailey, "Comparative tolerability profiles of oral antidiabetic agents," Drug Safety, vol. 11, no. 4, pp. 223-241, 1994.

[37] C. S. Harris, L. P. Beaulieu, M. H. Fraser et al., "Inhibition of advanced glycation end product formation by medicinal plant extracts correlates with phenolic metabolites and antioxidant activity," Planta Medica, vol. 77, no. 2, pp. 196-204, 2011.

[38] C. S. Harris, M. Asim, A. Saleem, P. S. Haddad, J. T. Arnason, and S. A. L. Bennett, "Characterizing the cytoprotective activity of Sarracenia purpurea L., a medicinal plant that inhibits glucotoxicity in PC12 cells," BMC Complementary and Alternative Medicine, vol. 12, article 245, 2012.

[39] R. Vianna, A. Brault, L. C. Martineau, R. Couture, J. T. Arnason, and P. S. Haddad, "In vivo anti-diabetic activity of the ethanolic crude extract of Sorbus decora C.K. Schneid. (Rosacea): a medicinal plant used by Canadian James Bay Cree Nations to Treat Symptoms Related to Diabetes," Evidence-Based Complementary and Alternative Medicine, vol. 2011, Article ID 237941, 7 pages, 2011.

[40] J. A. Guerrero-Analco, L. Martineau, A. Saleem et al., "Bioassayguided isolation of the antidiabetic principle from Sorbus decora (Rosaceae) used traditionally by the Eeyou Istchee Cree First Nations," Journal of Natural Products, vol. 73, no. 9, pp. 1519$1523,2010$.

[41] H. M. Eid, A. Brault, M. Ouchfoun, F. Thong, D. Vallerand, and L. Musallam, "Lingonberry (vacciniumvitis-idaea L) mobilizes L6 muscle GLUT4 transporters and exerts anti-obesity and antidiabetic effects in vivo," Austin Journal of Endocrinology and Diabetes, vol. 1, p. 11, 2014.

[42] H. M. Eid, L. C. Martineau, A. Saleem et al., "Stimulation of AMP-activated protein kinase and enhancement of basal glucose uptake in muscle cells by quercetin and quercetin glycosides, active principles of the antidiabetic medicinal plant vaccinium vitis-idaea," Molecular Nutrition and Food Research, vol. 54, no. 7, pp. 991-1003, 2010.

[43] H. M. Eid, M. Ouchfoun, A. Brault et al., "Lingonberry (vaccinium vitis-idaea L.) exhibits antidiabetic activities in a mouse model of diet-induced obesity," Evidence-Based Complementary and Alternative Medicine, vol. 2014, Article ID 645812, 10 pages, 2014.

[44] J. Capeau, "Insulin resistance and steatosis in humans," Diabetes and Metabolism, vol. 34, no. 6, pp. 649-657, 2008. 
[45] M. M. Ollmann and G. S. Barsh, "Down-regulation of melanocortin receptor signaling mediated by the amino terminus of Agouti protein in Xenopus melanophores," The Journal of Biological Chemistry, vol. 274, no. 22, pp. 15837-15846, 1999.

[46] C. Di Lorenzo, M. Dell'agli, M. Badea et al., "Plant food supplements with anti-inflammatory properties: a systematic review (II)," Critical Reviews in Food Science and Nutrition, vol. 53, no. 5, pp. 507-516, 2013.

[47] J. A. Cabrera Medaglia and C. López Silva, Addressing the Problems of Access: Protecting Sources, While Giving Users Certainty, IUCN, 2007.

[48] G. Burton and E. A. Evans-Illidge, "Emerging R and D law: the Nagoya Protocol and its implications for researchers," ACS Chemical Biology, vol. 9, no. 3, pp. 588-591, 2014. 


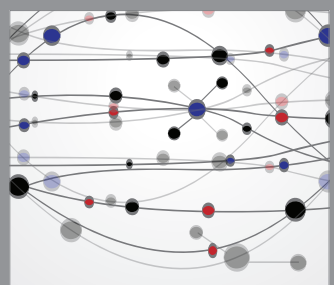

The Scientific World Journal
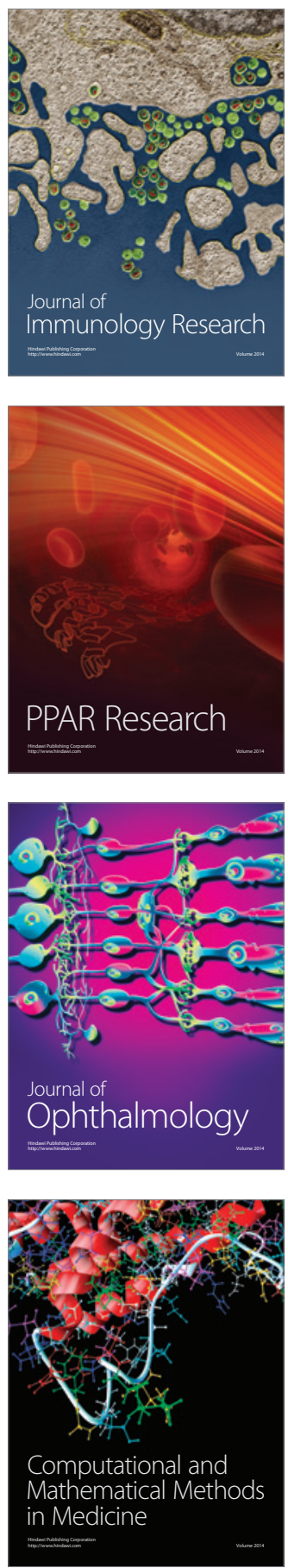

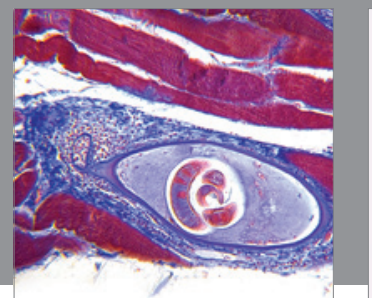

Gastroenterology

Research and Practice
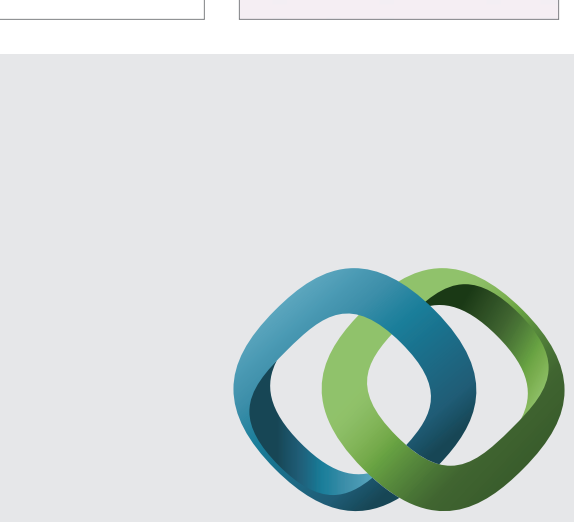

\section{Hindawi}

Submit your manuscripts at

http://www.hindawi.com
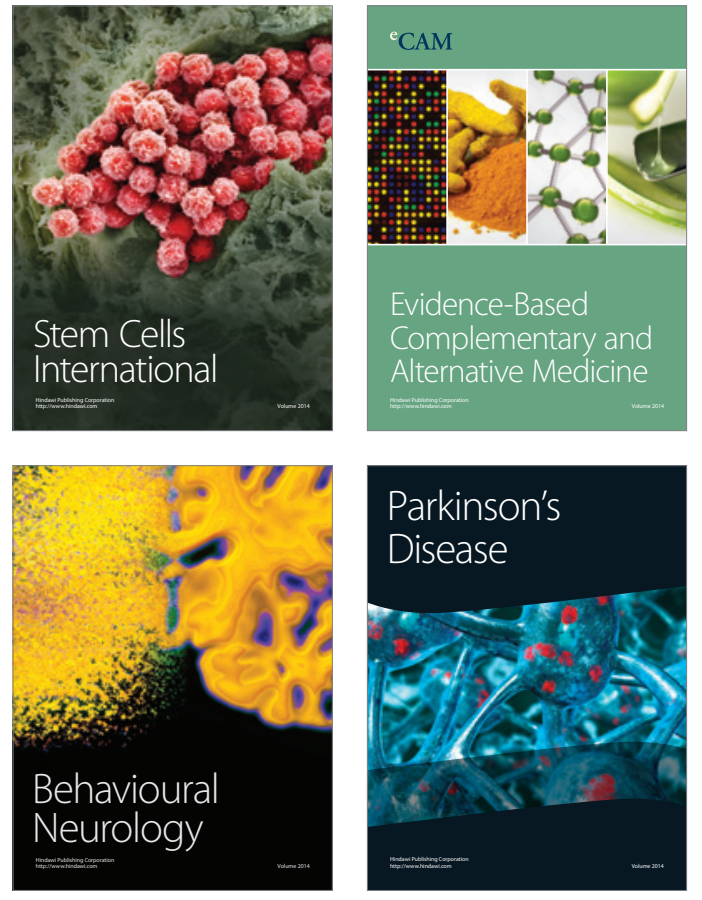
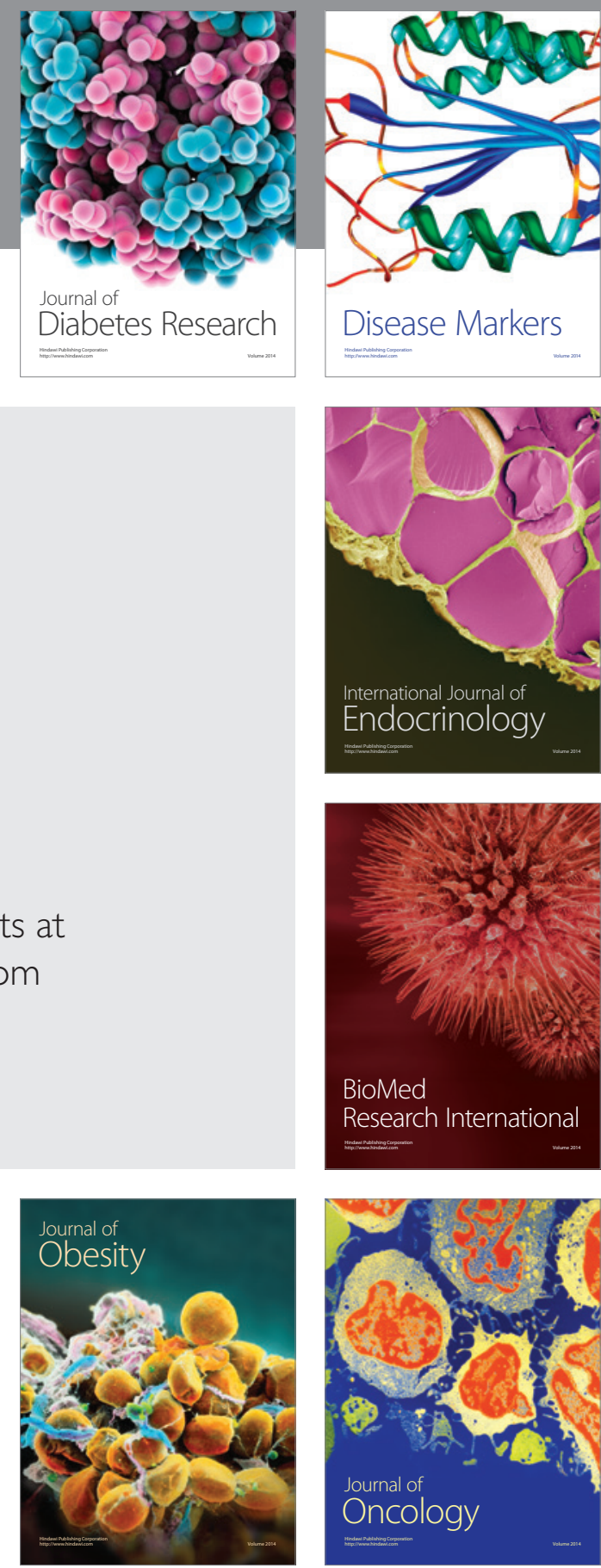

Disease Markers
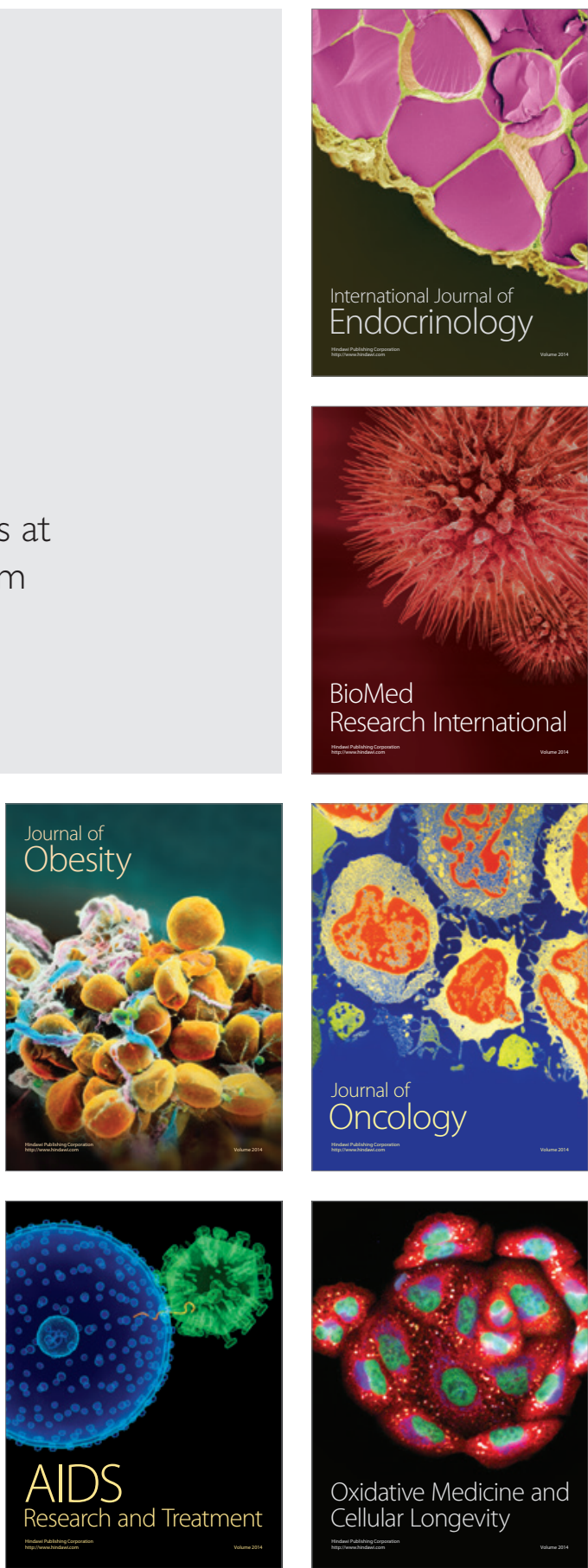